\title{
A Wideband Directional Microstrip Slot Antenna for On-Body Applications
}

\author{
Mehdi Hamidkhani*, Behdad Arandian \\ Department of Electrical Engineering, Dolatabad Branch, Islamic Azad University, Isfahan, Iran
}

Email address:

mehdi.hamidkhani@gmail.com (M. Hamidkhani), b.arandian@yahoo.com (B. Arandian)

To cite this article:

Mehdi Hamidkhani, Behdad Arandian. A Wideband Directional Microstrip Slot Antenna for On-Body Applications. Journal of Electrical and Electronic Engineering. Vol. 3, No. 3, 2015, pp. 48-51. doi: 10.11648/j.jeee.20150303.15

\begin{abstract}
Antennas are one of the main components of every wireless telecommunication system. With wideband antennas for on-body applications, there are some additional features that should be considered including radiation and physical size. In this paper, we presented a wideband omnidirectional slot antenna with a reflector element improvised under the feed line in order to minimize the impact of body on the antenna. Curiously, this reflector element allows for directionality of the antenna. In the following sections of the paper we will show that less power can penetrate body tissues in the presence of reflector elements; thus, wideband directional antennas are influenced less by human body than omindirectional antenna.
\end{abstract}

Keywords: Directivity, On-Body Application, Slot Antenna, Reflector Element, Wideband

\section{Introduction}

There may not be direct transmission of microwave signals in the human body, because they encounter significant losses there. However, wideband signals may be used for the transmission of narrow pulses with little multipath fading and interference, which are present in signal frequency communication channels. One of the requirements of the on-body installations of communication systems is their low transmission power, so that no adverse radiation effects are incurred on the human body. Consequently, there is a need for the development of wideband antennas with appropriate near and far field radiation characteristics for the on-body application in body-centric wireless communication systems [1-4].

For the installation of lower power transmitter and receiver equipment on the human body or clothing, the near field radiation of antennas inside or in the vicinity of human body should be considered. Also the dissipation of RF power inside the human body should be minimized. Furthermore, the antenna far field radiation should be considered for the communication among the on-body sensors and among these sensors and among these sensors and some nearby equipment (such as a mobile phone or a laptop personal computer). Consequently, the wideband antennas should be designed according to the specified characteristics $[2,5,6]$.

In this paper, we present a novel wideband slot antenna
[7-10] for on-body installation, which may be applied in low power communication systems to provide a very low radiation power towards the human body and an appropriate directional for field radiation pattern. The criterion for the wideband impedance bandwidth is $\mathrm{SWR}<2.5$ for the frequency band 6-10 GHz. An appropriate circuit is devised for the impedance matching at the antenna feed. Due to the complex interaction between an on-body antenna and the human body, we use full-wave numerical electromagnetic solvers, such as HFSS and FEKO.

\section{The Proposed Antenna Structure}

We use the substrate Rogers 5880 with dielectric constant $\epsilon_{r}=2.2$, height $\mathrm{h}=1.6 \mathrm{~m}$ and loss tangent $\tan \delta=0.0009$. Two U-shaped slots are made on the ground plane on one side of the substrate with the dimensions shown in figure 1 . The feed line (with characteristic impedance $50 \Omega$ ) is branched into two symmetrical strips (of impedance $100 \Omega$ ) on its other side. This feed configuration provides wideband matching for slotted and stacked patches. Such a slot antenna provides an omnidirectional pattern in the $\mathrm{XZ}$ plane. In order to reduce the radiation on the feed line side of the antenna, a narrow rectangular conducting plane as a reflector is placed at a distance $\mathrm{h}$ from it. 


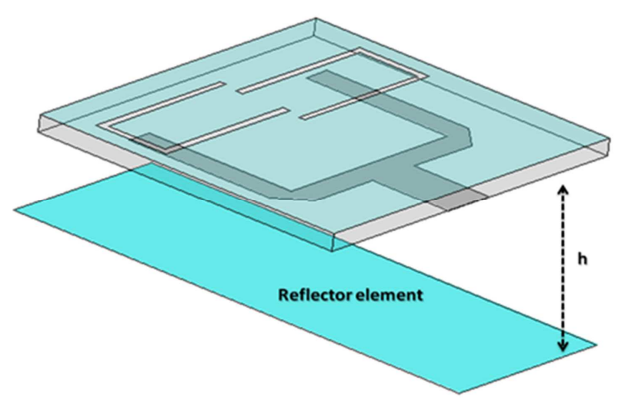

(a)

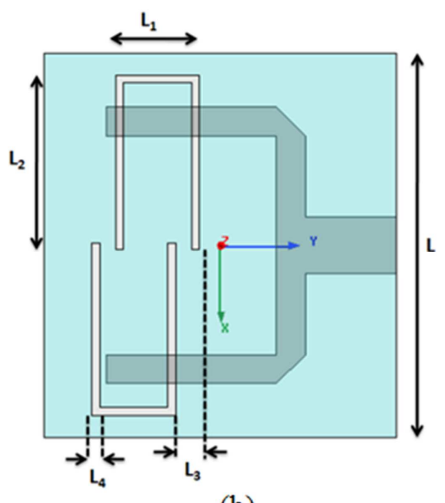

(b)

Figure 1. Proposed antenna configuration; (a) 3d view, (b) top view.

\section{Computer Simulation of Isolated Antenna}

The proposed antenna is designed and optimized by the full-wave simulation software HFSS. The simulation results and measurement data for SWR of the slot antenna without the reflector plane are shown versus frequency in figure 2 . The frequency band for $\mathrm{SWR} \leq 2.5$ is $5.2-10 \mathrm{GHz}$. Now consider a reflector plane as a rectangular conductor plate of dimensions $16 \mathrm{~mm} * 44 \mathrm{~mm}$ placed under the feed line at a distance $\mathrm{h}=3 \mathrm{~mm}$. Its SWR is drawn in figure 3, where the frequency band is $5.6-10.3 \mathrm{GHz}$ for $\mathrm{SWR} \leq 2.5$. The radiation patterns of the antenna without and with the reflector plate are drawn in figure 4 , at three frequencies 6,8 and $10 \mathrm{GHz}$, respectively. The antenna gains with and without reflector plate are drawn in figure 5 . It can be seen that the gain of the antenna with the reflector plane is relatively higher, which makes it a directional antenna.

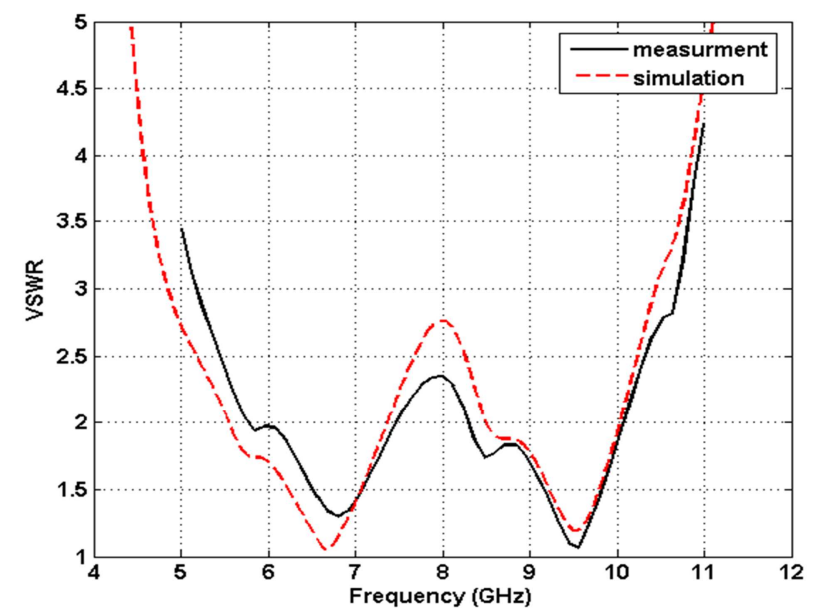

Figure 2. Simulated and measured VSWRs of proposed antenna without the reflector plane. The geometrical parameters are $L=32 \mathrm{~mm}, L 1=6.9 \mathrm{~mm}$, $L 2=14.4 \mathrm{~mm}, L 3=2 \mathrm{~mm}, L 4=0.6 \mathrm{~mm}$.

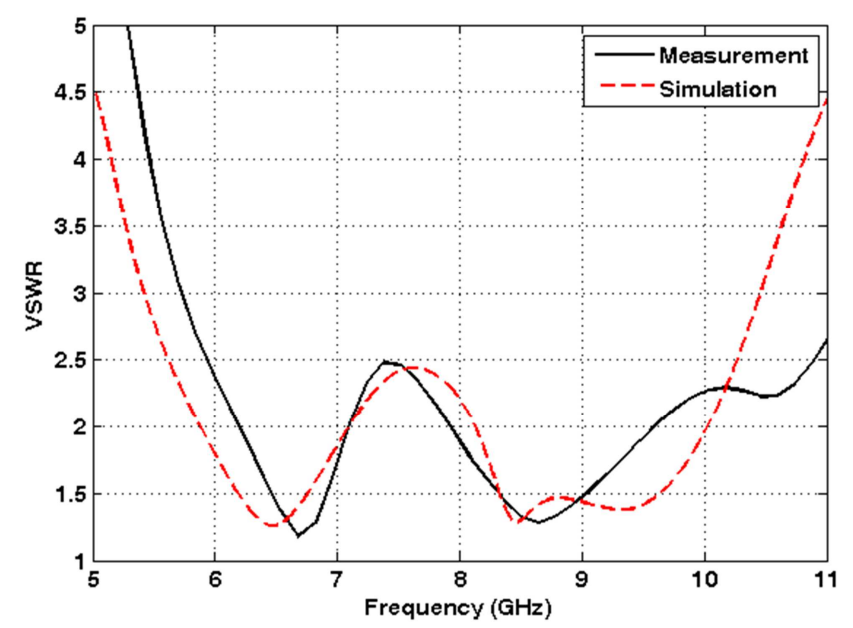

Figure 3. Simulated and measured VSWRs of proposed antenna with the reflector plane.

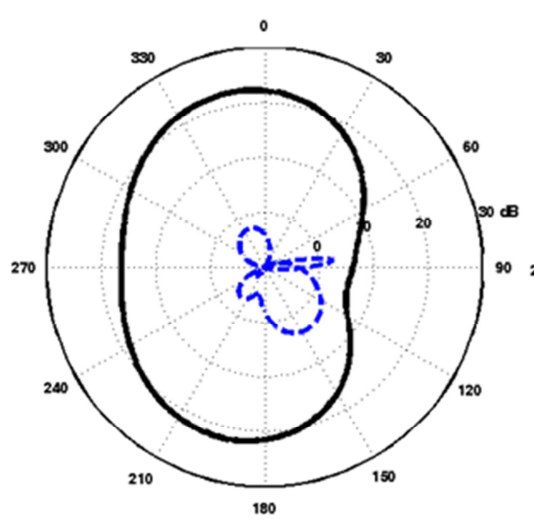

$\mathrm{XZ}$ plane at $6 \mathrm{GHz}$

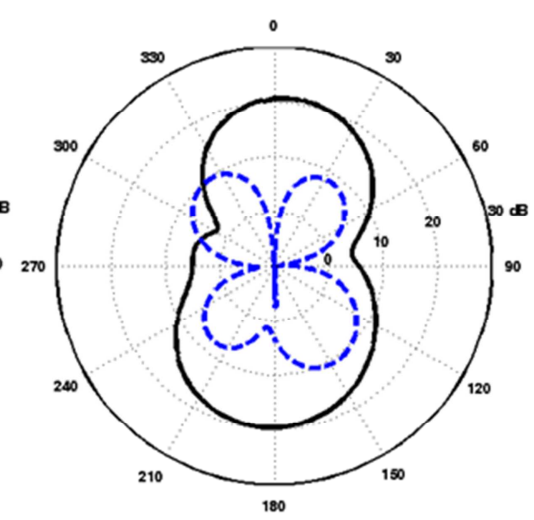

$\mathrm{XZ}$ plane at $8 \mathrm{GHz}$

(a)

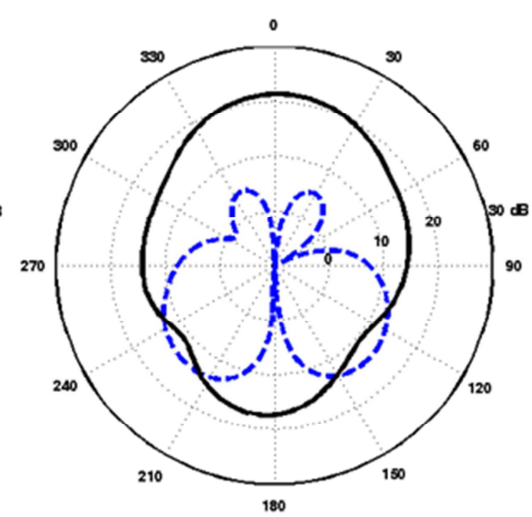

$\mathrm{XZ}$ plane at $10 \mathrm{GHz}$ 


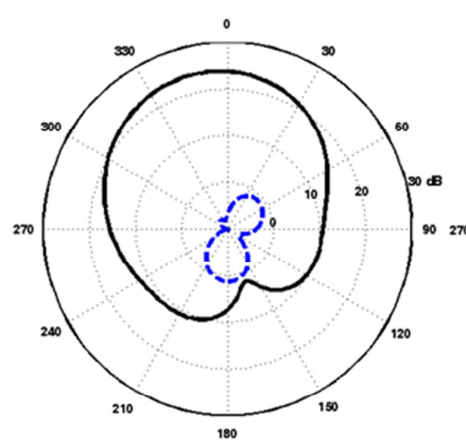

XZ plane at $6 \mathrm{GHz}$

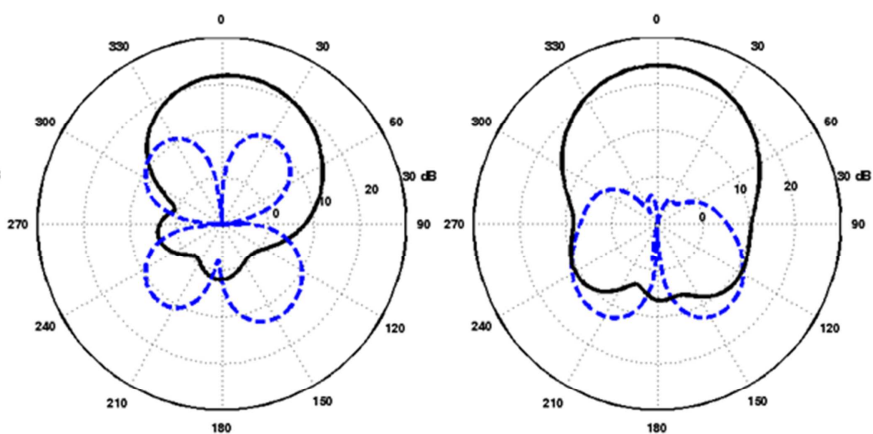

XZ plane at $8 \mathrm{GHz}$

(b)

\section{$E_{\varphi}-$ Solid line \\ $E_{\theta} \ldots$ Dotted line}

Figure 4. Measured radiation patterns of the proposed antenna in the XZ plane; (a) without the reflector plate, and (b) with the reflector plate.

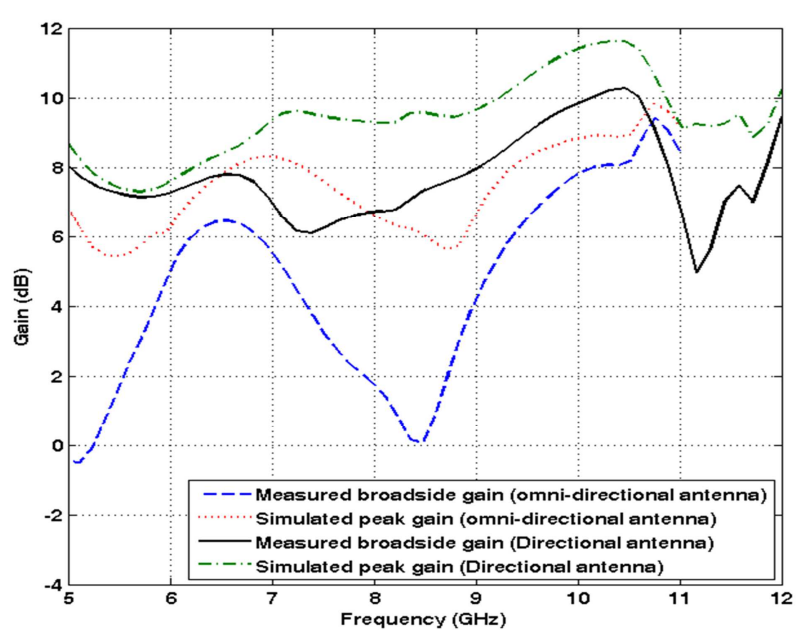

Figure 5. Simulated and measured gain of the proposed antenna.

\section{Computer Simulation of the On-Body Antenna}

We may consider two simplified models for the operation of the on-body antenna as given in [2]:

1- A three tissue model consisting of three layers of skin layer (with thickness $1 \mathrm{~mm}$ ), fat layer (with thickness $3 \mathrm{~mm}$ ) and muscle layer (with thickness $40 \mathrm{~mm}$ );

2- A homogenous model composed of a muscle layer (of thickness $44 \mathrm{~mm}$ ) with dielectric constant $\epsilon_{r}=52.7$, conductivity $\sigma=1.773$ and loss tangent $\tan \delta=0.242$.

The dimensions of both models are $120 \times 110 \times 44 \mathrm{~mm}^{3}$. The size of this simplified model is obtained by computer simulation of on-body antennas. For simplicity we use the second model. First, place the antenna feed line (without the reflector plate) at a distance of $3 \mathrm{~mm}$ from the body. In figure 6(a) performances of the isolated antenna and on-body antenna is compared based on their SWR versus frequency. We can note that the bandwidth for $\mathrm{SWR} \leq 2.5$ of the on-body antenna is quite narrower than that of the isolated antenna.
Next, place the antenna with the reflector plate on the body muscle tissue. Its SWR is drawn in figure 6(b) and compared with that of the isolated antenna. Based on the figure the SWR versus frequency curve behave similarly at higher frequencies, but are somewhat different at lower frequencies. Curiously, these lower frequencies lie outside the working frequency of the antenna. Consequently, the reflector plate behaves as an effective shield to protect the body against exposure to the antenna radiations.

This is because less power can penetrate body tissues in the presence of the reflector elements. In general, wideband directional antenna is influenced less by human body than omnidirectional antenna. In addition, changes observed for omnidirectional antenna during proximity to human body is dependent upon its distance from the body. More specifically, when small-size wideband antennas are studied in proximity to body models (reactive near field), the results rely the size of the antenna and its distance from the body. However, orientation is not necessarily a good solution for on-body antennas. The reason is that orientation of the antenna is measured in far field, while near fields of the antenna is not accounted for. Even if a directional antenna is placed in a specific angle, the near fields can measure great in the direction of human body.

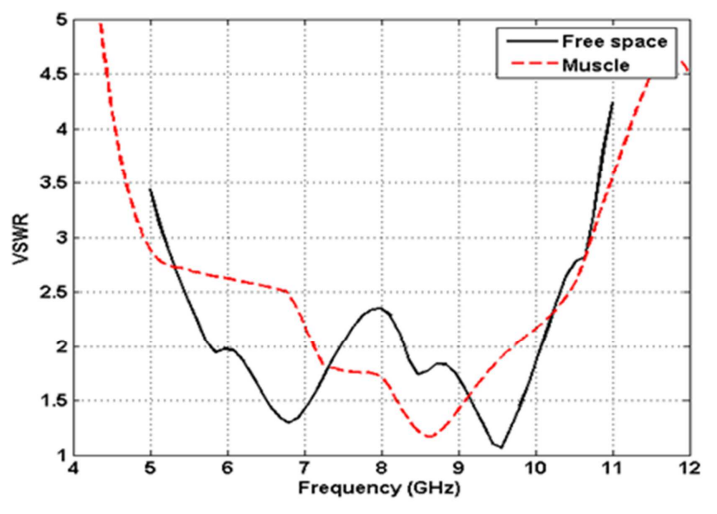

(a) 


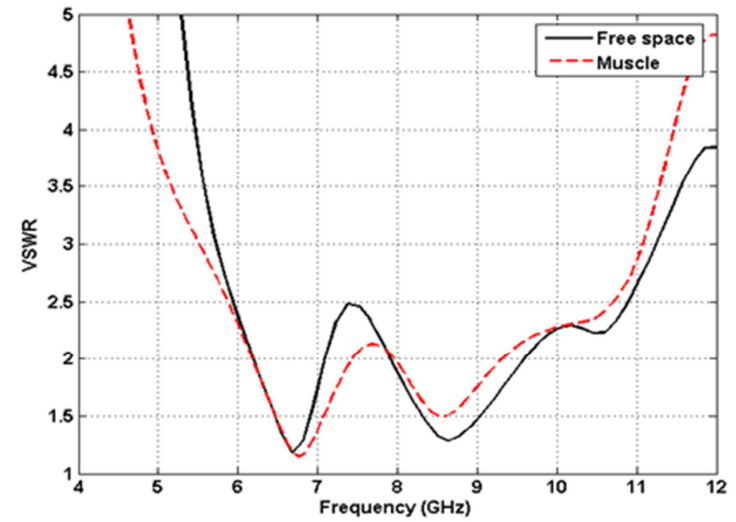

(b)

Figure 6. Simulated VSWR of the isolated antenna and on-body antenna; (a) without the reflector plate; (b) with the reflector plate.

\section{Conclusion}

In this paper we presented a wideband omnidirectional slot antenna. In order to minimize the impact of body on the antenna, a reflector element was improvised under the feed line. In addition, further directionality and orientation was achieved in the presence of this reflector element. We concluded that the wideband directional antenna is influenced less by proximity to human body than the omnidirectional antennas. Furthermore, changes observed for the omnidirectional antenna during proximity to human body is dependent upon its distance from the body.

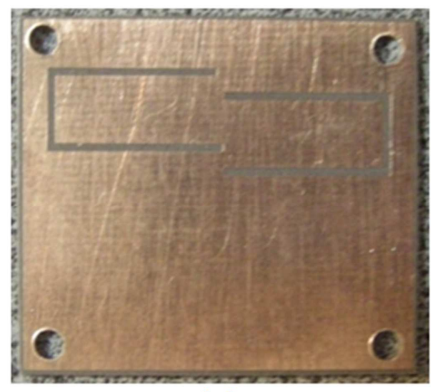

(a)

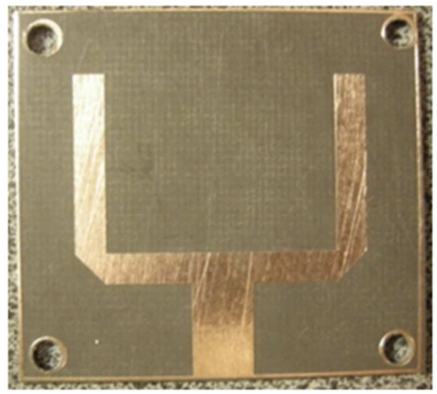

(b)

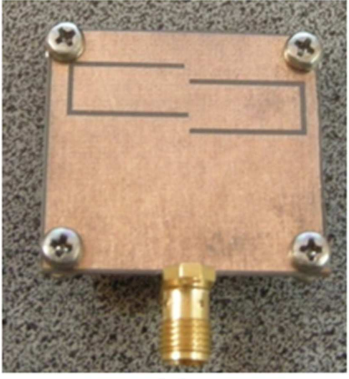

(c)

Figure 7. Images of the fabricated antenna; (a) top view, (b) bottom view, (c) $3 d$ view.

\section{References}

[1] Klemm, M.; Kovcs, I.Z.; Pedersen, G.F.; Troster, G., "Novel small-size directional antenna for UWB WBAN/WPAN applications," Antennas and Propagation, IEEE Transactions on, vol.53, no.12, pp.3884, 3896, Dec. 2005.

[2] Peter S.Hall, and Yang Hao, "Antenna and Propagation for Body-Centric Wireless Communications" Artech House Publisher, 2006, ISBN 1-58053-493-7, pp. 93-109.

[3] Cai, A.; See, T.S.P.; Zhi Ning Chen, "Study of human head effects on UWB antenna," Antenna Technology: Small Antennas and Novel Metamaterials, 2005. IWAT 2005. IEEE International Workshop on, vol., no., pp.310, 313, 7-9 March 2005.

[4] Oppermann, I., M. Hamalainen, and J. Iinatti, (eds.), UWB: Theory and Applications, New York: John Wiley \& Sons, 2004.

[5] Alomainy, A.; Hao, Y.; Hu, X.; Parini, C.G.; Hall, P.S., "UWB on-body radio propagation and system modelling for wireless body-centric networks," Communications, IEE Proceedings- , vol.153, no.1, pp.107,114, 2 Feb. 2006.

[6] K. Zhao, S. Zhang, Z. Ying, T. Bolin, and S. He, "Reduce the Hand-Effect Body Loss for LTE Mobile Antenna in CTIA Talking and Data Modes," Progress In Electromagnetics Research, PIER 137, 73-85, 2013.

[7] Balanis, C. A., Antenna Theory: Analysis and Design, 3rd ed., New York: Wiley-Interscience, 2005.

[8] Seong-Youp Suh; Stutzman, W.L.; Davis, William A., "A new ultrawideband printed monopole antenna: the planar inverted cone antenna (PICA)," Antennas and Propagation, IEEE Transactions on, vol.52, no.5, pp.1361, 1364, May 2004.

[9] Alomainy, A.; Hao, Y.; Parini, C.G.; Hall, P.S., "Comparison between two different antennas for UWB on-body propagation measurements," Antennas and Wireless Propagation Letters, IEEE , vol.4, no., pp.31,34, 2005.

[10] Samal, P.B.; Soh, P.J.; Vandenbosch, G.A.E., "UWB All-Textile Antenna With Full Ground Plane for Off-Body WBAN Communications," Antennas and Propagation, IEEE Transactions on , vol.62, no.1, pp.102,108, Jan. 2014. 